\title{
Numerical Calculation of Energy Between the Sealing Materials and Sepiolite Crystal on the Different Directions
}

\author{
Kun $\mathrm{Li}^{1, \mathrm{a}}$, Boqin $\mathrm{Gu}^{2, \mathrm{~b}}$ and Wanfu $\mathrm{Zhu}^{2, \mathrm{c}}$ \\ ${ }^{1}$ College of Mechanical Engineering, Anhui University of Science and Technology, Huainan 232001 \\ People's Republic of China; \\ ${ }^{2}$ School of Mechanical and Power Engineering, Nanjing Tech University, Nanjing, 211800 People's \\ Republic of China. \\ alikun46@163.com, bkli@njtech.edu.cn, cwfzhu@aust.edu.cn
}

Keywords: Energies, Nitrile-Butadiene Rubber, Sepiolite, Numerical Calculation.

\begin{abstract}
Using Universal field under a NVE ensemble Numerical Calculation and simulations were performed to investigate the interface binding energies between the Nitrile-Butadiene Rubber(NBR) and sepiolite fibers on (-1 $\left.\begin{array}{lll}-1 & 0\end{array}\right)$ and $\left(\begin{array}{lll}1 & 0 & 0\end{array}\right)$ lattice plane respectively. The result shows that the total force is repulsive force except the force between the acrylonitrile and NBR in the $\left(\begin{array}{lll}-1 & 0 & 0\end{array}\right)$ lattice plane. And the result reveals that the repulsive force of NBR/butadiene is much greater than NBR/acrylonitrile which indicates that acrylonitrile is easier to bind with NBR than butadiene. At the same time there is the conclusion that the electrostatic force is dominant comparing VDW and there is no chemical bond and hydrogenbond force.
\end{abstract}

\section{Introduction}

Interface bonding strength of the fiber and matrix are often subject to a large number of primary formula, surface treatment and content design, and then a large number of the measured surface and the mechanical properties of screening, the consumption of things take time.

In addition, for micromechanics testing is difficult, the interface between the fiber surface and matrix strength of different test methods will be the result of the difference is very big. Although there are a lot of from micro mechanics, mechanics of interface, the Nitrile Rubber (Nitrile-Butadiene Rubber, NBR) as the matrix, in a variety of Short Fiber to enhance body of Short Fiber Reinforced composite material (Short - Fiber - Reinforced Composites, SFRC) is analyzed with mechanics, numerical simulation, etc.[1], but many mechanics coefficient of interface are mostly through some mechanics model set, did not reach the interface combination between the essence of the problem [2].

Closely related to force and the interface binding energy, starting from the interface binding energy research interface force is an effective method. In recent years, using molecular dynamics (MD) simulation to research the composition of alloy materials, the relationship between structure and properties for exploration and development [3, 4]。Especially for the mechanical properties of carbon nanotubes/polymer composite materials interface combination and so on, a considerable amount of research[5-8] Thanks to the carbon nanotubes are small, the model is suitable for calculation of MD sepiolite fiber composite materials composed of MD simulation in connection to the atomic number is too much, not suitable for the whole model is set up.

In order to reduce the amount of calculation, this paper regarded the sepiolite as substrate, the use of a handful of NBR molecular chain to study the different combination of sepiolite in the face of the combination of performance, thus for the study of seal, the mechanical properties of composite material interface and failure analysis.

\section{Force Field, Model and Calculation}

\subsection{Modeling and Simulating.}

First use the Accelrys company Materials Studio package Visualizer module, real nitrile rubber are two butadiene and acrylonitrile monomer by emulsion polymerization and random copolymer, 
molecular chain as shown in fig. 1(a), in order to study the combination of butadiene and acrylonitrile performance respectively, construct the degree of polymerization of 20 butadiene and acrylonitrile single molecule chains, as shown in fig 1.(b), (c).Input sepiolite crystal model, as shown in fig1(d).

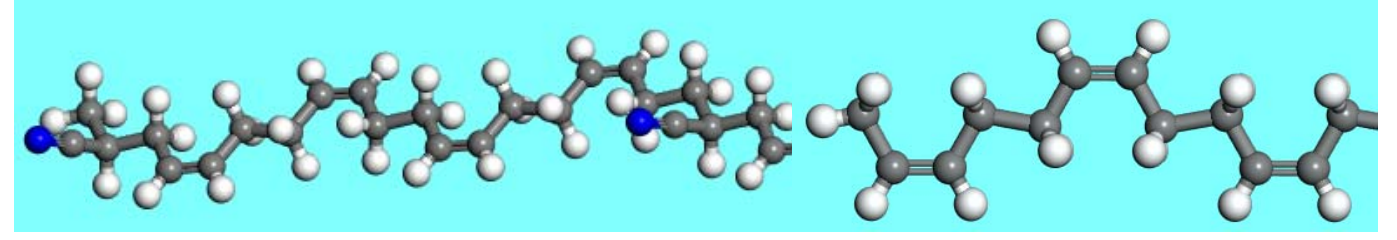

(a) NBR one section in the single molecule

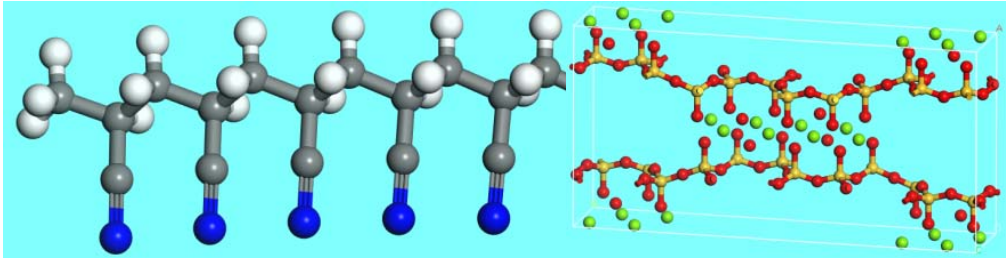

(c) acrylonitrile molecule chain

(d) sepiolite crystal cell

Fig. 1 initial molecular model building (white on behalf of the $\mathrm{H}$ atoms; gray on behalf of the $\mathrm{C}$ atoms; blue stands for $\mathrm{N}$ atoms; red represents $\mathrm{O}$ atoms; green for $\mathrm{Mg}$ atoms; yellow for $\mathrm{Si}$ atoms)

Then use the Visualizer module build interface system is shown in figure 2.

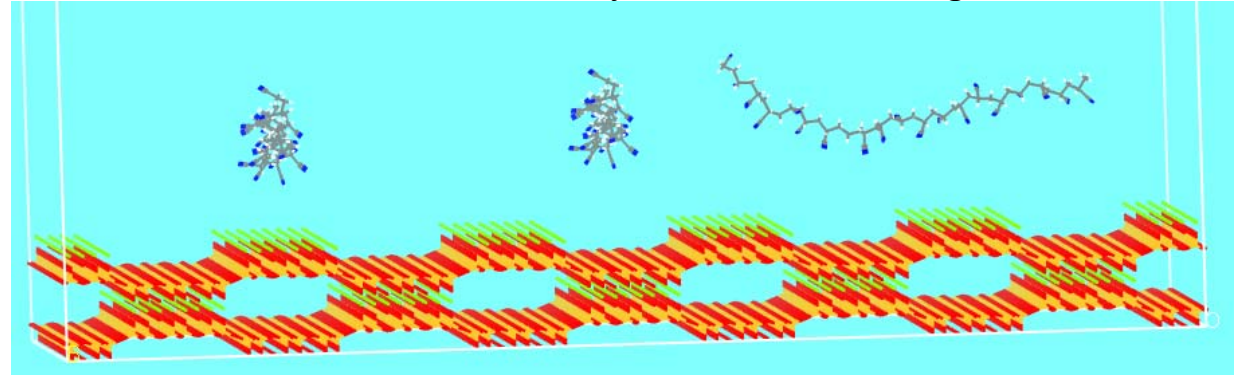

Fig. $2\left(\begin{array}{lll}1 & 0 & 0\end{array}\right)$ crystal plane of acrylonitrile with initial configurations

Use the Minimizer and Dynamics in the Discovery modules, in COMPASS [9], choose NVT ensemble under the force field, the temperature set at $320 \mathrm{k}, \mathrm{MD}$, cyclical simulation research.As defined by the Maxwell - the Boltzman distribution of initial Velocity of atomic motion, the integral using Verlet Velocity method, the Time step of $1 \mathrm{fs}$, Dynamics, Time of $10 \mathrm{ps,} \mathrm{simulate} \mathrm{the}$ temperature control in the process of using the method of Anderson [10], van der Waals (VDW) and electrostatic interactions (Coulomb) using Atom - -based and Ewald method respectively, truncation radius of $9.5 \times 10-10 \mathrm{~m}$, calculated at HP Z820 workstations to complete.Curves of temperature in the process of calculation with the simulation time and energy with the volatility of the simulation time curve as shown

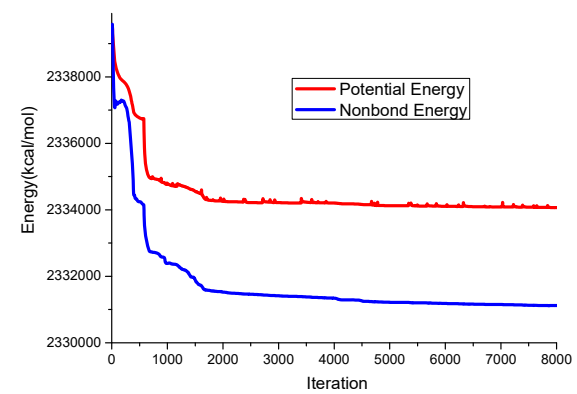

(a) On the face $\left(\begin{array}{lll}1 & 0 & 0\end{array}\right)$ of butadiene in

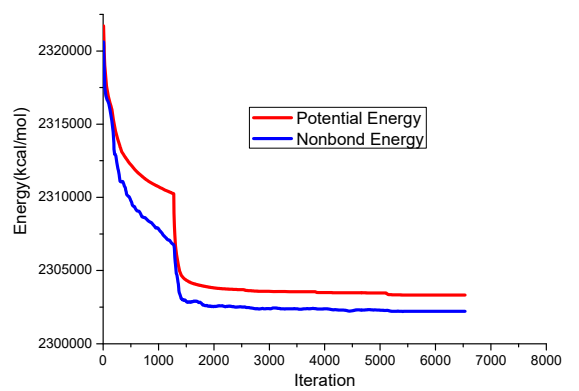

(b) On the face ( $\left.\begin{array}{lll}1 & 0 & 0\end{array}\right)$ acrylonitrile Fig. 3 Minimizer energy in the process of change 


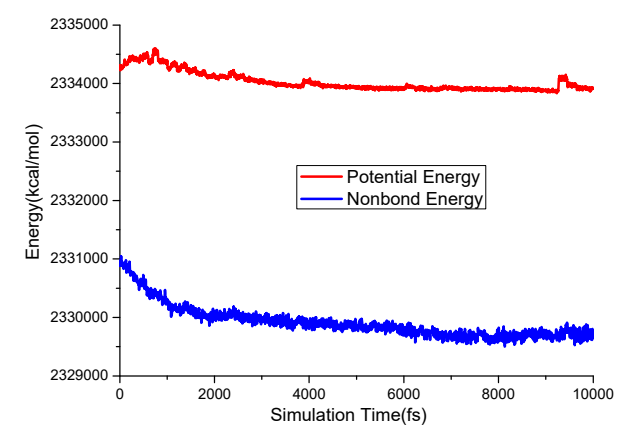

Fig. 4 Energy vs. simulation time

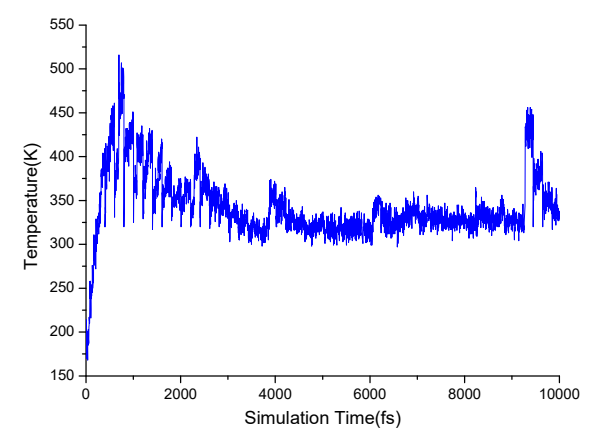

Fig. 5 Temperature vs. simulation time

Fig 6. is running after the MD of butadiene and sepiolite interface configuration, can be seen from the graph, after a full MD calculation, butadiene and sepiolite close together.

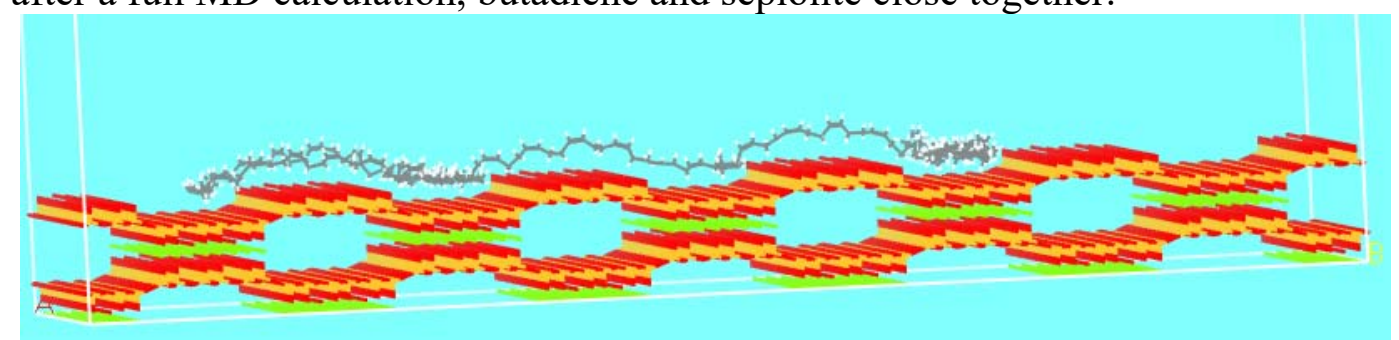

Fig. $6(1,0,0)$ and butadiene crystal after MD calculation of configurations

\subsection{Analyzing the simulation results.}

Define combination for interaction can be negative.Interaction energy of the composite material structure is equal to the balance of the total energy minus get rid of this structure after the energy of sepiolite, after subtracting get rid of sepiolite under the structure of nitrile rubber chain of energy, namely:

$$
E_{\text {Bind }}=-E_{\text {Inter }}=-\left(E_{\text {Total }}-E_{\text {Sepiolite }}-E_{\text {Molecule }}\right)=E_{\text {Sepiolite }}+E_{\text {Molecule }}-E_{\text {Total }}
$$

The binding energy is a sign of compound strength of interaction between components.Binding energy, the greater the said the stronger the interaction between the components, the formation of the greater the stability of the system, the interface between the reaction might have, the greater the more able to withstand external static load and dynamic load.

Butadiene, acrylonitrile and sepiolite interface binding energy is expressed as two crystal direction $E_{\text {Bind/Butadiene }}^{-100}, \quad E_{\text {Bind/Acrylonitrile }}^{-100}, E_{\text {Bind/Butadiene }}^{100}, \quad E_{\text {Bind/Acrylonitrile }}^{100}$

The results showed that:

$E_{\text {Bind/Acrylonitrile }}^{-100}=785.22>0$, attraction;

$E_{\text {Bind/Butadiene }}^{-100}=-27071.56<0$, repulsive force;

$E_{\text {Bind/Butadiene }}^{100}=-32005.97<0$, repulsive force;

$E_{\text {Bind/Acrylonitrile }}^{100}=-7224.96<0$, repulsive force;

And there are:

$$
\begin{aligned}
& \left|E_{\text {Bind/Butadiene }}\right| \gg\left|E_{\text {Bind/Acrylonitrile }}\right| \\
& \left|E^{-100}\right|<\left|E^{100}\right|
\end{aligned}
$$

(1) Show that acrylonitrile as polar molecules and the combination of sepiolite has a better effect;Type 2 shows that the genesis of magnesium ion concentration on offshore bubble areas between the binding energy, the greater the this can also be attested from the figure, figure of acrylonitrile in nitrogen had crossed the sepiolite crystal cell wall into magnesium enrichment region.

The result is in the case of fixed sepiolite all atoms results, aims to study different crystal direction, respectively, and the size of the different components form the binding energy of nitrile rubber, nitrile rubber foam and actual Shanghai mutually binding energy of gravity and large (otherwise).This is 
mainly because the fixed sepiolite magnesium atoms is fixed, oxygen activation energy, prevents the mg ions into the rubber molecules, and so cannot be a huge caused by electrostatic force.

\section{Summary}

(1) Different directions of nitrile rubber on the molecular force is not the same, close to one side of the magnesium binding energy. Can get out to sea foam filling, reinforcing fiber as nitrile rubber, rely mainly on the activation energy of magnesium in rubber molecules to form larger binding force.

(2) The combination of acrylonitrile and sepiolite have better sex, acrylonitrile content, the more you can join more sepiolite as filler.

(3) Compared to the van der Waals force, electrostatic force in combining dominant role in the field of force, no chemical bond force and hydrogen bond force.

\section{References}

[1]. Fu, Y. Molecular Dynamics Simulation on Binding Energies and Mechanical Properties of HTPB and Different Crystal Faces of Al. ACTA PHYSICO-CHIMICA SINICA, 2009. 25(1): p. 187-190.

[2]. Zhang, B., B. Gu and X. Yu, Failure behavior of resorcinol-formaldehyde latex coated aramid short-fiber-reinforced rubber sealing under transverse tension. Journal of Applied Polymer Science, 2014. 132(4167212).

[3]. Shi, S., S. Zhang and Q. Zhang, Probing Difference in Binding Modes of Inhibitors to MDMX by Molecular Dynamics Simulations and Different Free Energy Methods. PLOS ONE, 2015. 10(e014140910).

[4]. Sahoo, B.R., et al., Exploration of the binding modes of buffalo PGRP1 receptor complexed with meso-diaminopimelic acid and lysine-type peptidoglycans by molecular dynamics simulation and free energy calculation. CHEMICO-BIOLOGICAL INTERACTIONS, 2014. 220: p. 255-268.

[5]. López-Manchado, M.A., et al., Dynamic mechanical and Raman spectroscopy studies on interaction between single - walled carbon nanotubes and natural rubber. Journal of Applied Polymer Science, 2004. 92(5): p. 3394-3400.

[6]. Zeng, Q.H., A.B. Yu and G.Q. Lu, Multiscale modeling and simulation of polymer nanocomposites. PROGRESS IN POLYMER SCIENCE, 2008. 33(2): p. 191-269.

[7]. Harishkumar, C. and R.H. Schuster, Influence of ACN Content on the Properties of NBR/CNT Nanocomposites. KGK-KAUTSCHUK GUMMI KUNSTSTOFFE, 2014. 67(3): p. 22-28.

[8]. Rouhi, S., Y. Alizadeh and R. Ansari, Molecular dynamics simulations of the single-walled carbon nanotubes/poly (phenylacetylene) nanocomposites. Superlattices and Microstructures, 2014. 72: p. 204-218.

[9]. Rahman, A., Molecular Dynamics Study of Liquid Water. The Journal of Chemical Physics, 1971. 55(7): p. 3336.

[10]. Levesque, D., L. Verlet and J. Kürkijarvi, Computer "Experiments" on Classical Fluids. IV. Transport Properties and Time-Correlation Functions of the Lennard-Jones Liquid near Its Triple Point. Physical Review A, 1973. 7(5): p. 1690-1700. 